\title{
Análisis de indicadores de aprendizaje organizacional: implicaciones en la mejora del instrumento de investigación
}

\author{
Mesinas Cortés Cesar¹, Mancinas González Abelardo², Quijano Vega Gil Arturo**
}

\begin{abstract}
${ }^{1}$ Departamento de Ciencias Económico Administrativas del Instituto Tecnológico de Hermosillo.
2 División de Estudios de Posgrado e Investigación del Instituto Tecnológico de Hermosillo.

Cuerpo académico: Competitividad Organizacional y Regional.
\end{abstract}

\section{Resumen}

La importancia de medir el aprendizaje organizacional en MiPyMes radica en la identificación de capacidades dinámicas distintivas, las cuales impactan en los niveles de innovación y competitividad. Al tratar de lograr esta actividad hemos identificado que el instrumento de investigación no siempre se ajustaba a la teoría. Por ello, el objetivo que se persigue es demostrar el grado de ajuste de los datos observados, a través de la implementación de dos cuestionarios, a un modelo hipotetizado de aprendizaje organizacional que pretende contestar ¿En qué medida la medición del aprendizaje organizacional en MiPyMes del sector alimentario de Hermosillo, Sonora, impacta los niveles de innovación y competitividad?

Los resultados encontrados demuestran que usando el método de ecuaciones estructurales, existen altos niveles de $\mathrm{KMO}$, de alfa de Cronbach, y mediana correlación. Esto como resultado de haber aumentado y reordenado, de acuerdo al modelo original, los ítems en el segundo cuestionario, así como haber aumentado el número de empresas encuestadas. En conclusión, las mejoras en el instrumento de investigación, dio como resultado tener mejor evidencia de haber identificado a las MiPyMes que han logrado aprendizajes organizacionales significativos impactando sus procesos de innovación y competitividad.

Palabras clave: Aprendizaje organizacional, Competitividad, Innovación.

\section{Legal reflections on the participation of civil society in the Regional Develop- ment Committees of Mining States and Municipalities in Mexico}

\begin{abstract}
The importance of measuring organizational learning in MiPyMes lies in the identification of distinctive dynamic capabilities, which impact innovation and competitiveness levels. In trying to achieve this activity we have identified that the research instrument did not always fit the theory. Therefore, the objective is to demonstrate the degree of adjustment of the observed data, through the implementation of two questionnaires, to a hypothetical model of organizational learning that aims to answer to what extent the measurement of organizational learning in MiPyMes del food sector of Hermosillo, Sonora, impacts the levels of innovation and competitiveness?

The results show that using the method of structural equations, there are high levels of $\mathrm{KMO}$, Cronbach's alpha, and median correlation. This as a result of having increased and rearranged, according to the original model, the items in the second questionnaire, as well as having increased the number of companies surveyed. In conclusion, the improvements in the research instrument resulted in having better evidence of having identified the MSMEs that have achieved significant organizational learning impacting their innovation and competitiveness processes.
\end{abstract}

Keywords: Organizational learning, innovation, competitiveness . 


\section{INTRODUCCIÓN}

En el marco de una economía del conocimiento (Powell y Snellman, 2004) identificamos el Aprendizaje Organizacional (AO) como el modelo empresarial que permite formar capacidades dinámicas distintivas, impactando positivamente a la innovación y competitividad (Ambec y col. 2013). Por ello, el $\mathrm{AO}$ es el proceso de medición del aprendizaje de conocimientos diversos, localizados en las habilidades y capacidades de diferentes actores. En ese sentido, el objetivo que se persigue es demostrar el grado de ajuste de los datos observados, a través de la implementación de dos cuestionarios, a un modelo hipotetizado de aprendizaje organizacional, que pretende contestar iEn qué medida la medición del aprendizaje organizacional en MiPyMes del sector alimentario de Hermosillo, Sonora, impacta los niveles de innovación y competitividad?

En Sonora existen poco más de 100 mil empresas, de las cuales, 5 mil pertenecen a la rama económica de alimentos (productoras y de servicios), de ese universo cerca de 2000 empresas están ubicadas en Hermosillo (DENUE, 2014). Al margen de las empresas grandes y empresas maquiladoras, las MiPyMes de la rama manufacturera y de servicios de alimentos en Hermosillo es la más importante por el número de empresas y empleos. Se estima que en los últimos 10 años, la rama de alimentos de la región ha sufrido diversos cambios organizacionales, ante la competencia que infringe los productos provenientes de otras partes del estado, del país y de los mercados globales (Mesinas, 2010).

En correspondencia, el método de ecuaciones estructurales (MES) elegido fue apropiado para medir si los ítems diseñados en un cuestionario, son apropiados y representativos para ajustarse a las exigencias de un modelo teórico. Esto es así porque MES, además de validar la confianza del instrumento de investigación, también confirma la validez del modelo, es decir, pone a prueba la teoría. Para ello, MES permite realizar un análisis factorial relacionando medidas de adecuación muestral de Kaiser-Meyer-Olkin (KMO); Test de esfericidad de Barlett, a través, de chi cuadrada con sus grados de libertad y significación. El objetivo del análisis factorial es definir cuántos factores explican la mayor parte de la varianza.

La relación entre aprendizaje organizacional y competitividad se fundamenta en las aportaciones iniciales de Jenkin (2013), Camisón (2002) y Real, Leal y Roldán (2006), los cuales coinciden en que el estudio de capacidades y habilidades (aprendizaje organizacional) es la base en los estudios de competitividad. Así, las diferentes áreas del conocimiento, localizadas en las organizaciones, explican el grado de avance en el aprendizaje e innovación frente a la competencia del mercado. Todo lo cual, justifica una metodología de tratamiento estadístico basado en MES.
Por otra parte, los estudios de AO se consideran prácticas emergentes dentro de los estudios de Competitividad. Las aportaciones de Fraj y col. (2015), Real y col. (2006), así como de Camisón (2002) coinciden al enfatizar que, ante la abrumadora expansión de los estudios de Competitividad empresarial, se había olvidado la importancia básica del AO en esos estudios. En resumen, a los estudios tangibles de Competitividad les hacen falta los estudios intangibles del AO, que son la raíz de la explicación de las competencias organizacionales. De esta forma el aprendizaje se convierte en un aspecto estratégico.

En relación a la competitividad, tomamos en cuenta el modelo de diamante de Porter (Allen y col. 2006) y especialmente la propuesta de competitividad del Banco Interamericano de Desarrollo (Saavedra, 2012) el cual incluye los siguientes indicadores: planeación estratégica, producción y operaciones, calidad, comercialización, contabilidad y finanzas, recursos humanos, gestión ambiental y sistemas de información.

En relación al modelo MES de ecuaciones estructurales como método de estudio estadístico podemos mencionar las observaciones que hacen Chatfield (2018), así como Alaminos y col. (2015), en relación a las grandes posibilidades de ajuste de los datos observables ante variables latentes no observables (constructos, factores). La ecuación representativa del modelo es $1 \mathrm{mk}=\beta \mathrm{m}+\tau €$ $+Z$, donde 1 es el valor total de ajuste entre variables, $m$ son las variables latentes endógenas y $\mathrm{k}$ las variables latentes exógenas; $\beta \mathrm{m}$ (beta) la matriz de dimensiones $\mathrm{m}$, de peso $\beta$ que determina la relación entre dos variables latentes endógenas; $\tau$ (gama) la matriz de dimensiones $m$, de peso $r$ que determinan la relación entre una variables endógena y una exógena, ambas latentes; $\epsilon$ (xi) vector de dimensiones $\mathrm{k} \times 1$, de variables latentes exógenas; $\mathrm{Z}$ (zeta) vector, de dimensiones $\mathrm{m} \times 1$ determinada por el grado de error.

En general, los resultados se relacionan con la prueba que se realiza a las hipótesis, específicamente a las variables y sus ítems. El ajuste del modelo de independencia, que asume la no relación entre los datos debe rechazarse, mientras que el promedio de los residuos entre la correlación/covarianza observada de la muestra y el modelo esperado, en la hipótesis alternativa debe aceptarse. El método de ecuaciones estructurales (MES) elegido mide si los ítems diseñados en un cuestionario, son apropiados y representativos para ajustarse a las exigencias de un modelo teórico. Esto es así porque MES, además de validar la confianza del instrumento de investigación, también confirma la validez del modelo, es decir, pone a prueba la teoría. Para ello, MES permite realizar un análisis factorial relacionando medidas de adecuación muestral de Kaiser-Meyer-Olkin (KMO); Test de esfericidad de Barlett, a través, de chi cuadrada con sus grados de libertad y significación. El objetivo del análisis 
factorial es definir cuántos factores explican la mayor parte de la varianza.

Para validar la fiabilidad del instrumento a partir del indicador alfa de Cronbach, que además toma en cuenta el grado de correlación entre variables e ítems, el procedimiento seguido fue el siguiente: se implementó un cuestionario de 72 preguntas que logró alto índice de alfa de Cronbach; al terminar la encuesta a 42 empresas se encontró la no existencia de correlación lineal, para salvar la investigación se exploró la técnica de bondad de ajuste a un conjunto de hipótesis, logrando establecer alta correlación intraclase, con ello se identificaron ítems y empresas donde se demuestra correlación entre aprendizaje organizacional y su impacto en la innovación y competitividad. Sin embargo, sólo en algunas empresas, por ello en la segunda parte del estudio se tuvo que redefinir el modelo teórico y el instrumento de investigación. En ese sentido, se logró altos índices de alfa de Cronbach así como altos índices al emplear MES. Con lo cual se pudo mejorar el instrumento de investigación y aumentar el número de casos empresariales con amplia relación entre aprendizaje organizacional e innovación y competitividad.

Los resultados muestran que usando el método de MES se encontraron altos niveles de $\mathrm{KMO}$, de alfa de Cronbach, y medianas correlaciones. Esto como resultado de haber ajustado (reordenación y aumento) los ítems del segundo cuestionario al modelo AO. En adición, se aumentó el número de empresas encuestadas. En conclusión, las mejoras en el instrumento de investigación dieron como resultado ampliar e identificar a las MiPyMes que han logrado aprendizajes organizacionales significativos impactando los procesos de innovación y competitividad.

\section{METODOLOGÍA}

Dado que el estudio se considera exploratorio, el método de selección muestral fue de tipo aleatorio por atributos: años de experiencia y nivel de ingresos anuales.

En cuanto a las actividades productoras y de servicios alimenticios estudiadas, a nivel de clase, fueron las siguientes:

Comida americana; Comida Mediterránea; Comida y bebida de la India; Almeja entera y almeja picada; Comida americana (hamburguesas y más); Comida italiana; Comida Japonesa; Hamburguesas; Helados; Mariscos; Suchi; Comidas regionales; Ensaladas y baguettes; Pastelería y alta repostería; Taquería; Ensaladas sándwiches sopas; Hielo; Pizza; Ensaladas, sándwiches, hamburguesas, paninis; Pasteles, bollos y pays; Hemoglobina en Polvo y Plasma en Polvo; Agua; Café; Helados, paletas, aguas frescas; vegetales listos para comer; Platillos; Coyotas. Molino harinero, Producción de quesos, panaderías, tortillerías.

En estas condiciones se procedió a realizar el análisis estadístico. Primero, se evaluó la validez del instrumento encontrando un valor de alfa de Cronbach de .962. Este resultado confirmó su fiabilidad y confianza. En relación a las correlaciones, se encontró la no existencia de correlación lineal. Ante esta heterogeneidad, se procedió a evaluar la bondad de ajuste a partir de dos hipótesis:

H0: Las variables que integran el $\mathrm{AO}$ se comportan en forma independiente en relación a los ítems.

$\mathrm{H} 1$ : Las variables que integran el $\mathrm{AO}$ se comportan en forma dependiente en relación a los ítems.

En ese sentido, se estableció un 5\% de error, encontrando una correlación intraclase de .962, con un valor de significación de 0 , como se muestra en la figura 1. Para confirmar este dato, se procedió a calcular una medida chi cuadrada encontrándose un valor de 675.5, frente a un valor de 90.5313 buscado en tablas de probabilidades para chi cuadrada. Ello, significó rechazar la hipótesis nula aceptando la hipótesis alternativa. Una vez confirmado la existencia de correlación intraclase, se procedió a identificar las variables y empresas que representaban altos y medianos grados de AO. Se concluye que el mejor desempeño aprendizajecompetitividad está en las pequeñas y medianas empresas con amplia experiencia, avalada por haberse sostenido en la competencia por largos periodos de tiempo. En ese contexto, se identificó a 16 empresas y 27 ítems como los escenarios más significativos, y, por tanto, más cercanos al modelo AO.

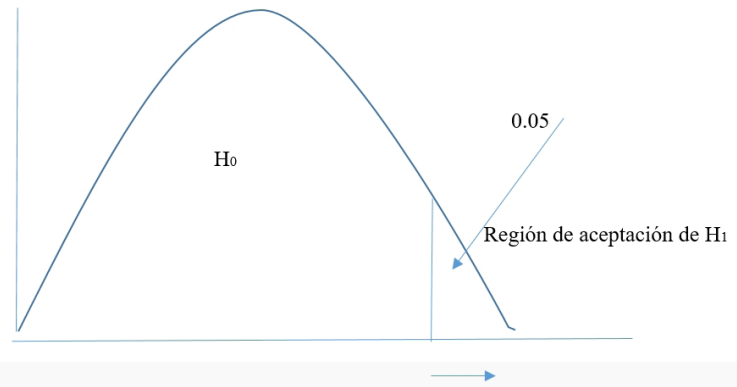

Fuente: elaboración propia.

Figura 1. Comparación de probabilidades basado en $X^{2}$

Debido a la reducción de empresas e ítems representativos de $\mathrm{AO}$, se inició otro estudio, el cual empezó por revisar y precisar el modelo AO. La relectura de los estudios de Ryan (2010) Real (2006), Camisón (2002) y Burstein y Vogel (2017), permitieron identificar otras variables importantes. Al modelo teórico utilizado le hacía falta 
incluir los conocimientos creados (CC) con anterioridad a la medición del $\mathrm{AO}$, sobre la base de que las empresas que han desarrollado deliberadamente sus procesos de aprendizaje tienen mejores conocimientos, por ello, tienen mayores posibilidades de innovar por encima de las empresas que no desarrollan sus procesos de aprendizaje. Otros aspectos considerados son la incorporación de ítems en la determinación de flujos feedforward y feedback, sin los cuales el modelo no encuentra explicación satisfactoria, como se ilustra en la figura 2.

Finalmente, de acuerdo a la relectura de a teoría de $\mathrm{AO}$ nos percatamos que la variable competitividad no es tomada en cuenta. A cambio, se replantea la variable rendimientos organizacionales (REN) en el sentido de combinar, bajo otro estilo de redacción, los ítems propios de REN y algunos de competitividad. Esto es así porque los estudios de AO representan buscar las raíces (aprendizajes y capacidades) que explican la competitividad de las empresas. De esta forma ha sido separada la variable competitividad.

En estas condiciones iniciamos el segundo estudio, con un instrumento que mide 8 variables de $\mathrm{AO}$ con 99 ítems: 5 ítems en conocimientos creados con anterioridad a la medición (CC), 9 en los aprendizajes logrados a nivel individual (AI), 10 en los aprendizajes logrados a nivel de grupo (AG), 15 en aprendizaje organizacional interno (AOI), 9 en aprendizajes logrados con el flujo feedforward (FF), 8 en aprendizajes logrados con el flujo feedback (FB), 18 en capacidades dinámicas distintivas (CDD), que permiten a la organización diferenciarse de la competencia por medio de la innovación, y 25 en rendimientos organizacionales equivalentes a la productividad y las rentas económicas (REND). También, se amplió la muestra a 83 empresas, sobre la base de encontrar más empresas medianas, pues sabemos que desarrollan deliberados procesos de aprendizaje (Mesinas, 2010). Enseguida se procedió al análisis estadístico, esta vez con un método diferente. Con el uso del modelo de ecuaciones estructurales se plantearon las siguientes hipótesis confirmatorias:

H1: Los conocimientos creados durante la creación y fomento de la empresa influyen positivamente en el aprendizaje organizacional.

H2: El aprendizaje organizacional como proceso de creación de conocimientos influye positivamente en el desarrollo de competencias dinámicas distintivas de la empresa.

H3: El aprendizaje organizacional como proceso de creación de conocimientos influye positivamente en el rendimiento organizacional de la empresa.

H4: Las competencias dinámicas distintivas influyen positivamente en el rendimiento organizacional de la empresa.

\section{RESULTADOS}

De acuerdo a los datos, según se muestra en la Tabla 1, la mayor parte de los constructos empleados en el cuestionario presentan altas medidas de adecuación muestral de $\mathrm{KMO}$ y altas medidas de test de esfericidad de Barlett. Sin embargo, AO_CC presenta bajo nivel de fiabilidad, mientras que AO_AO; AO_FB; AO_CDD y AO_REND están por debajo de la media de correlación de Pearson. Lo cual nos indica que algunos ítems aún faltan por rediseñar en el aspecto lingüístico, para que MES los identifique bajo una estructura

Figura 2. Modelo de gestión del aprendizaje y el conocimiento para el logro de la competitividad organizacional.

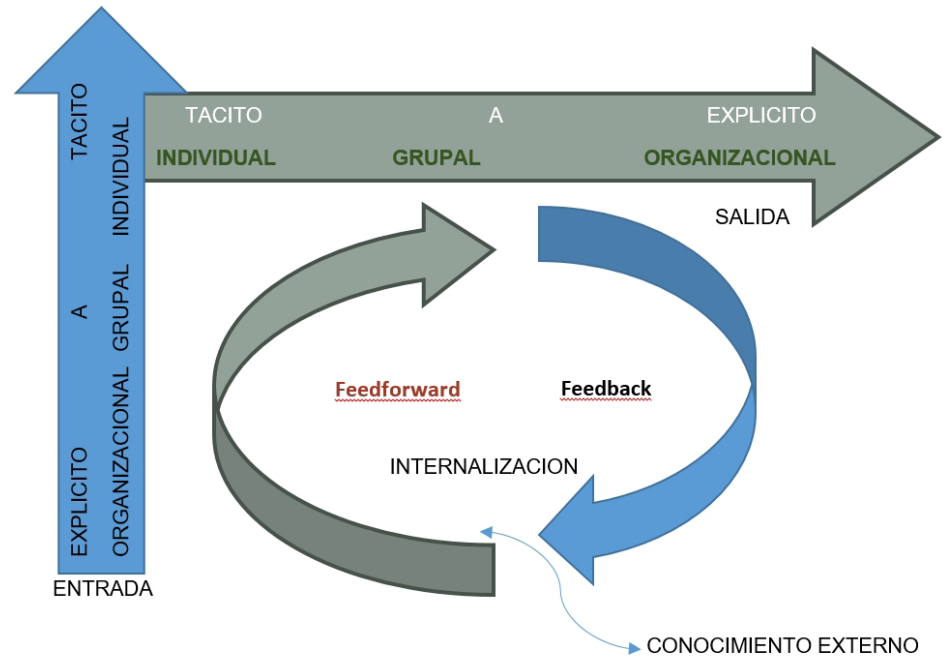

Fuente: adaptado de Crossan y Berdrow (2003), p.1091. 
lógica de coherencia y concordancia. Aunque los altos índices de KMO y Barlett permiten fiarnos de su validez. En MES se mide el grado de representación entre variables latentes endógenas.

Tabla 1. Validación del cuestionario final: calidad de precisión entre constructos-métrica-validación

\begin{tabular}{|c|c|c|c|c|}
\hline Constructo & Items & $\begin{array}{l}\text { Alfa de } \\
\text { Cronbach }\end{array}$ & Correlación & $\begin{array}{l}\text { Análisis } \\
\text { factorial }\end{array}$ \\
\hline AO_CC & 5 & .299 & .578 & $\begin{array}{l}\text { KMO }=.843 \\
\text { CHI CUADRADA = } 159 \\
\text { gl=15 } \\
\text { Significación }=000\end{array}$ \\
\hline AO_AI & 9 & .882 & .510 & $\begin{array}{l}\text { KMO }=.820 \\
\text { CHI CUADDADA }=320.3 \\
\text { gl }=36 \\
\text { Significación }=000\end{array}$ \\
\hline AO_AG & 10 & .894 & .515 & $\begin{array}{l}\mathrm{KMO}=.813 \\
\text { CHI CUADDADA =380.69 } \\
\text { gl=45 } \\
\text { Significación }=000\end{array}$ \\
\hline AO_AO & 15 & .887 & .427 & $\begin{array}{l}\text { KMO }=.837 \\
\text { CHI CUADRADA }=646.6 \\
\text { gl=105 } \\
\text { Significación }=000\end{array}$ \\
\hline AO_FF & 9 & .601 & .599 & $\begin{array}{l}\text { KMO=.600 } \\
\text { CHI CUADDRADA =106.35 } \\
\text { gl =36 } \\
\text { Significación = } 000\end{array}$ \\
\hline AO_FB & 8 & .717 & .410 & $\begin{array}{l}\text { KMO }=.717 \\
\text { CHI CUADDRADA =96.98 } \\
\text { gl }=28 \\
\text { Significación }=000\end{array}$ \\
\hline AO_CDD & 18 & .907 & .391 & $\begin{array}{l}\text { KMO }=.747 \\
\text { CHI CUADRADA =1014.1 } \\
\text { gl=143 } \\
\text { Significación }=000\end{array}$ \\
\hline AO_REND & 28 & .942 & .448 & $\begin{array}{l}\text { KMO =.874 } \\
\text { CHI CUUARADA =1462 } \\
\text { gl }=300 \text { No0 } \\
\text { Significación = } 000\end{array}$ \\
\hline
\end{tabular}

Así, se confirma que las hipótesis sometidas a comprobación y se acepta su validez, por lo que se confirma la validez del modelo. Cada hipótesis, y la variable en cuestión, representa la vinculación existente en la ecuación $1=\beta 8$ $+\tau €+Z$. Por ejemplo, en relación al AO_Al tenemos la siguiente ecuación: $1=.820+.18-.0025$ lo cual significa que .820 de Bao_ai explica alta vinculación entre el AO y el Aprendizaje Individual; .18 representa el valor de la relación entre $\mathrm{AO}$ como variable exógena y $\mathrm{Al}$ como variable endógena; - .0025 significa el grado de error al valorar el modelo MES. Todo lo cual influye en la determinación de validez de constructos y modelo teórico.

${ }^{1}$ La correlación es significativa en el nivel 0.01 ; chi cuadrada es significativa al 0.05; en AO_CC al menos tres factores explican el $60.1 \%$ de la varianza; en AO_Al al menos cinco factores explican el $72 \%$ de la varianza; en $\mathrm{AO}$ Al al menos 5 factores explican el $85.4 \%$ de la varianza; en OG_AG al menos siete factores explican el $85.69 \%$ de la varianza; en AO_CDD al menos 8 de los factores explican el $87.87 \%$ de la varianza; en $\mathrm{AO} \_\mathrm{FF}$ al menos cuatro factores explican el $68.86 \%$ de la varianza; en $\mathrm{AO} \_\mathrm{FB}$ al menos tres factores explican el $62 \%$ de la varianza; en AO_AREND al menos cuatro factores explican el $68.2 \%$ de la varianza.
El análisis factorial de los datos permitió definir a la mayoría de los factores explicados por sus ítems, porque explican más del $50 \%$ de la varianza. De acuerdo a la matriz de componentes fueron excluidos 26 ítems, y en función de la la matriz de componentes rotados se identificaron 57 interacciones con un coeficiente de concordancia de 69.9. De acuerdo a estimaciones tipo Tukey de potencia deben realizarse observaciones en las 8 variables para conseguir aditividad con un valor promedio de 3.546, dado un coeficiente de correlación intraclase de .942. En la estructura de factores se observa invarianza, esto es, aproximadamente las mismas varianzas en los ítems propuestos.

Otros resultados asociados al estudio estadístico tienen que ver con la identificación de empresas que aprenden a gestionar sus conocimientos mejorando sus niveles de innovación y competitividad. Todas las empresas estudiadas aprenden y se sitúan en el nivel que la gerencia establece. Las empresas con visión y objetivos de largo plazo son las que aprenden mejor. A ello, habría que agregar que planear a largo plazo significa asignar inversiones que apoyen permanentemente actividades en la formación de capacidades e intervenir en la innovación. En ese contexto, las empresas medianas se desenvuelven casi sin problemas para desarrollar el AO. El problema de empresas micro y pequeñas puede ser su falta de planeación estratégica, esto es, no tienen visión y objetivos de largo plazo, sin embargo, eso puede deberse a la falta de inversiones, siendo éste el factor que limita el avance en el AO.

Lo antes expuesto, puede explicar que más del 30\% de las empresas estudiadas no ofrecieron alta evidencia de AO. Algunas micro y pequeñas empresas, una vez incorporadas ciertas rutinas de trabajo, deciden quedarse en ese nivel. En cambio, el $70 \%$ son identificadas con altos y medianos aprendizajes. Se identifica que el comportamiento organizacional dirigido a obtener el conocimiento explícito, traducido en la obtención de conocimientos básicos como competencias, permite el logro de capacidades dinámicas distintivas, traducidas en capacidades de innovación y desarrollo de procesos y productos, esto es, la obtención del conocimiento tácito. Todo lo cual es correlativo de una Planeación estratégica previamente implantada.

Los beneficios del modelo incluyen rentabilidad que permite sostener las actividades en el corto y mediano plazo, y en algunos casos en el largo plazo. La suma de capacidades básicas, más las capacidades dinámicas distintivas, permiten un grado de competitividad que permite sostenerse en el mercado con éxito.

Según los items identificadas con alta validez, permiten señalar que los estilos de aprendizaje de las pequeñas empresas del sector alimenticio de Hermosillo, desarrollan diversos procesos de exploración o feedforward y de 
explotación o feedback, donde la organización impacta el aprendizaje de grupos de trabajo y éstos a los trabajadores.

\section{CONCLUSIONES}

Las características del estudio fueron de tipo exploratorio. Aunque se ha avanzado en la mejora del instrumento de investigación, y de acuerdo a los datos arrojados en la matriz de componentes y de componentes rotados, se debe comprender que la mejora de un instrumento de investigación es una actividad permanente. En este caso, recomendamos modificaciones del modelo teórico $\mathrm{AO}$ en a pego al cambiante contexto regional de las MiPyMes.

En ese sentido, la experiencia lograda ha sido importante, pues pocas veces se hacen este tipo de estudios en la localidad, frente a los abundantes estudios, en la materia, que se hacen de empresas grandes y maquiladoras. Esperamos entregar los resultados a las empresas encuestadas para que las empresas identifique el modelo $\mathrm{AO}$ y lo adopten como práctica cotidiana.

Así mismo, consideramos necesario que las empresas formalicen esas condiciones en un modelo de AO, que permita medir sus activos de aprendizaje y conocimientos y sus impactos en la competitividad de forma permanente. En el presente estudio, hemos logrado comparar dos escenarios, a partir de los resultados de las encuestas, poniendo a prueba la probabilidad de que exista independencia entre variables, contra la probabilidad de la presencia de una relación de dependencia entre las mismas. Estadísticamente se comprueba la validez del segundo escenario.

Identificamos, en la última muestra, que todas las empresas forman capacidades básicas de aprendizaje explícito, que estas condiciones iniciales influyen positivamente en el siguiente eslabón de aprendizaje: la formación de capacidades dinámicas distintivas, que son el reflejo, de grupos expertos al interior y al exterior de las empresas que permiten realizar innovación y desarrollo de procesos, productos y servicios tales como:

- Trazabilidad en actividades alimenticias.

- Mejora de nuevos procesos que reducen costos y mejoran la calidad del producto.

- La producción de alimentos tradicionales con atributos nuevos.

- La diversificación productiva.

- La capacitación de personas y grupos al logro de conocimientos tácitos (planeación, logística, nuevas tecnologías, nuevos equipos y maquinaria, publicidad y mercadotecnia sobre escenarios virtuales y electrónicos).

\section{BIBLIOGRAFÍA}

Alaminos, A. y Francés, F. Penalva-Verdú, C. y Santacreu, O. 2015. Análisis multivariante para las ciencias sociales I. Pyldos Ediciones. Cuenca, Ecuador.

Allen, R. y Helms, M. 2006. Linking strategic practices and organizational performance to Porter's generic strategies. Business Process Management Journal. 12: 433-454.

Ambec, S., Cohen, M.A, Elgie, S. y Lanoie, P. 2013. The Porter Hypothesis at 20: Can Environmental Regulation Enhance Innovation and Competitiveness? Review of Environmental Economics and Policy. 7: 2-22.

Burstein, A. y Vogel, J. 2017. International Trade, Technology, and the Skill Premium Journal of Political Economy. 125: 1356-1412.

Camisón, C. 2002. Las competencias distintivas basadas en activos intangibles. Ch. 6 en Nuevas claves para la dirección estratégica P. Morcillo, y J. Fernández, (Ed), pp.117-151. Ariel, Barcelona.

Chatfield, Ch. 2018. Introduction to Multivariate Analysis. Routledge. New York.

Crossan, M. y Berdrow, I. 2003. Organizational Learning and Strategic Renewal. Estrategic Management Journal. 24:1087-1105.

DENUE.2014. Directorio Estadístico Nacional de Unidades Económicas. INEGI. https://www.inegi.org.mx/app/ mapa/denue/

Fraj, E., Matute, J. Melero, I. 2015. Environmental strategies and organizational competitiveness in the hotel industry: The role of learning and innovation as determinants of environmental success. Tourism Management. 46: 3042.

Jenkin. T.A. 2013. Extending the 4 I Organizational Learning Model: Information Sources, Foraging Processes and Tools. Administrative Sciences. 3: 96-109.

Mesinas, C. 2010. Gestión del Conocimiento: medición del aprendizaje manufacturero en pymes de Hermosillo, Sonora. Universidad de Málaga-EUMED. Málaga.

Powell, W. y Snellman, K. 2004. The Knowlwdgw Economy. Annu. Rev. Sociol. 30: 199-220.

Real, J.C., Leal, A. y Roldan, J.L. 2006. Aprendizaje organizativo y tecnologías de la información. Revista Europea de Dirección y Economía de la Empresa. 15: 9-32.

Ryan, T. 2010. Driving Technical Change. Pragmatic Bookshelf. Dallas.

Saavedra García, M.L. 2012. Una propuesta para la determinación de la competitividad en la pyme latinoamericana. Pensamiento y Gestión. 33: 93-124. 